\title{
Illuminating the dynamics of biofilms
}

\author{
Suzanne C. Letham and Tanmay A. M. Bharat
}

This month's Under the Lens discusses dual-view light-sheet microscopy and how its use has revealed the dynamics of bacterial biofilm development, a fundamental process found in bacteria.

Bacteria are typically thought of as singlecelled planktonic organisms, free-floating in the environment. However, most bacteria exist in macroscopic multicellular communities called biofilms. This multicellular lifestyle of bacteria is often overlooked, despite being essential to bacterial survival. Resolution limitations of conventional confocal microscopy have thus far prevented lineage and trajectory mapping of individual micron-sized bacterial cells within densely packed and actively growing biofilms. Therefore, the developmental dynamics of biofilm formation are not fully understood. Here, we highlight a study that utilized advanced optical microscopy to map trajectories of single cells in Vibrio cholerae biofilms, starting from a single founder cell to a mature multicellular biofilm, providing novel insights into biofilm formation."

To track individual bacterial cells within dynamic $V$. cholerae biofilms, Qin et al., developed a novel dual-view light-sheet microscopy ${ }^{2,3}$ workflow, which enabled isotropic spatial resolution to be obtained with an order of magnitude lower photobleaching compared to spinning disk confocal microscopy. Light-sheet microscopy enables live imaging of biological samples by illuminating a sheet of light on the sample orthogonal to the imaging axis, illuminating only the plane in focus, thereby limiting the dose suffered by the specimen. Dual-view or multi-view light-sheet microscopy allows simultaneously illumination and imaging of the sample from more than one direction, providing different views of the sample. Combining the data from these different views creates a volume with isotropic resolution in all three dimensions. This novel microscopy workflow facilitated the spatial resolution of individual bacterial cells over time during biofilm growth. Fluorescent labelling of cells was performed using a constitutively expressed avian reovirus protein that formed distinct punctae. These punctae were inherited during cell division, allowing optical sectioning limitations associated with light sheet microscopy to be overcome. After cell division, the daughter cell formed a new punctum, facilitating lineage tracing, and allowing cell trajectories to be mapped during biofilm formation. The trajectories of cells in the $V$. cholerae biofilms were consequently followed for several hours at high temporal resolution.

The application of this imaging approach has provided new insights into biofilm formation. The study showed that in the early stages of biofilm formation, cells grew laterally displaying random Brownian motion, with cells that formed early in biofilm development becoming trapped at the substrate. Vertical, ballistic growth was observed after 7 hours, with a 'fountain-like flow' of cells pushing cells to the front of the biofilm, away from the substrate. Comparing mutants with wild-type $V$. cholerae biofilms revealed that the 'fountain-like flow' of cells required the extracellular biofilm matrix protein $\mathrm{RbmA}$, suggesting that it has a key role in uniform lateral biofilm expansion.

The use of such novel microscopy techniques to study cell trajectories in biofilm formation are rapidly advancing our knowledge of this fundamental, yet poorly understood,

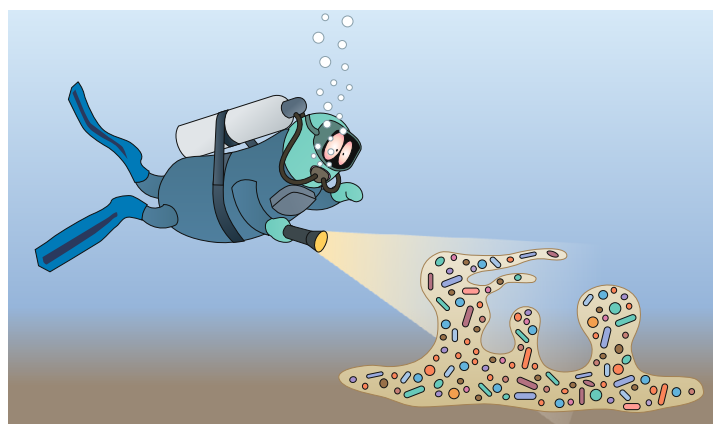

Credit: Philip Patenall/Springer Nature Limited

prokaryotic developmental process. Data generated by such high-volume microscopy methods are revolutionizing the field by feeding into and driving computational modelling approaches ${ }^{4}$. Better models will lead to a step change in our ability to predict biofilm dynamics under a variety of conditions. This will advance our understanding of the fundamental principles of bacterial biofilm growth from an initial single, planktonic cell to a multicellular community. Sir William Dunn School of Pathology, University of Oxford, Oxford, UK.

凶e-mail: underthelens@bioch.ox.ac.uk

https://doi.org/10.1038/s41579-020-00439-x

1. Qin, B. et al. Cell position fates and collective fountain flow in bacterial biofilms revealed by light-sheet microscopy. Science 369, 71-77 (2020).

2. Keller, P. J. et al. Reconstruction of zebrafish early embryonic development by scanned light sheet microscopy. Science 322, 1065-1069 (2008).

3. McDole, K. et al. In toto imaging and reconstruction of post-implantation mouse development at single-cell level. Cell 175, 859-876 (2018).

4. Hartmann, R. et al. Emergence of three-dimensional order and structure in growing biofilms. Nature Physics 15, 251-256 (2019).

Competing interests

The authors declare no competing interests. 